\title{
Global Hopf Bifurcation Analysis for a Time-Delayed Model of Asset Prices
}

\author{
Ying Qu and Junjie Wei \\ Department of Mathematics, Harbin Institute of Technology, Harbin 150001, China \\ Correspondence should be addressed to Junjie Wei, weijj@hit.edu.cn
}

Received 7 October 2009; Accepted 13 January 2010

Academic Editor: Xuezhong He

Copyright (C) 2010 Y. Qu and J. Wei. This is an open access article distributed under the Creative Commons Attribution License, which permits unrestricted use, distribution, and reproduction in any medium, provided the original work is properly cited.

A time-delayed model of speculative asset markets is investigated to discuss the effect of time delay and market fraction of the fundamentalists on the dynamics of asset prices. It proves that a sequence of Hopf bifurcations occurs at the positive equilibrium $v$, the fundamental price of the asset, as the parameters vary. The direction of the Hopf bifurcations and the stability of the bifurcating periodic solutions are determined using normal form method and center manifold theory. Global existence of periodic solutions is established combining a global Hopf bifurcation theorem with a Bendixson's criterion for higher-dimensional ordinary differential equations.

\section{Introduction}

Efficient Market Hypothesis (EMH) is a standard theory of financial market dynamics. According to the theory, asset prices follow a geometric Brownian motion representing the fundamental value of the asset, and hence asset prices cannot deviate from their fundamental values. The EMH theory, however, cannot explain excess volatility of financial markets such as speculative booms followed by severe crashes. Recently, models have been developed to explain fluctuations in financial markets (see [1-9] and the references therein). In such models, asset prices follow deterministic paths that can deviate from fundamental values generating what is called a speculative bubble in asset markets. In speculative markets modeling, almost all the previous models have utilized either differential or difference equations methodology.

Dibeh [4] presents a delay-differential equation to describe the dynamics of asset prices. The author divides market participants into chartists and fundamentalists. The fundamentalists follow the EMH theory and base their demand formation on the difference between the actual price of the asset $p$ and the fundamental price of the asset $v$. The chartists, on the other hand, base their decisions of market participation on the price trend of the asset. 
They attempt to exploit the information of past prices to make their decisions of purchasing or selling the asset. In [4], the model describing the time evolution of the asset price is given by

$$
\frac{\mathrm{d} p}{\mathrm{~d} t}(t)=(1-m) \tanh (p(t)-p(t-\tau)) p(t)-m(p(t)-v) p(t)
$$

where $m \in(0,1]$ is the market fraction of the fundamentalists. A time delay is introduced for chartists since they base their estimation of the slope of the asset price trend on an adaptive process that takes into account past values of the slope of the price trend.

In [4], it is shown by simulation that there may exist limit cycles for $(\mathrm{P})$, explaining the persistence of deviations from fundamental values in speculative markets. The aim of the present paper is to provide a detailed theoretical analysis of this phenomenon from the bifurcation point of view. Applying the local Hopf bifurcation theory (see [10]), we investigate the existence of periodic oscillations for (P), which depends both on time delay $\tau$ and the market fraction of the fundamentalists $m$. Using the normal form theory and center manifold theorem [11, 12], we derive sufficient conditions for determining the direction of Hopf bifurcation and the stability of the bifurcating periodic solutions. Furthermore, taking $\tau$ as a parameter, we establish the existence of periodic solutions for $\tau$ far away from the Hopf bifurcation values using a global Hopf bifurcation result of $\mathrm{Wu}$ (see [13] and also [1418]). A key step in establishing the global extension of the local Hopf branch at the first critical value $\tau=\tau_{0}$ is to verify that (P) has no nonconstant periodic solutions of period $4 \tau$. This is accomplished by applying a higher-dimensional Bendixson's criterion for ordinary differential equations given by Li and Muldowney [19].

The rest of this paper is organized as follows: in Section 2, our main results are stated and some numerical simulations are carried out to illustrate the analytic results. In Section 3, results on stability and bifurcations at positive equilibrium are proved, taking $m$ and $\tau$ as parameters, respectively. In Section 4, a theorem on the direction and stability of Hopf bifurcation is provided. Finally, a global Hopf bifurcation theorem is proved.

\section{Main Results}

Given the nonnegative initial condition

$$
p(t)=\varphi \geq 0, \varphi(0)>0, \quad t \in[-\tau, 0]
$$

Equation $(\mathrm{P})$ admits a unique solution (Hale and Lunel [10]). Any solution $p(t, \varphi)$ of $(\mathrm{P})$ is nonnegative if and only if $\varphi(0)>0$, following the fact that

$$
p(t)=\varphi(0) e^{\int_{0}^{t}[(1-m) \tanh (p(s)-p(s-\tau))-m(p(s)-v)] \mathrm{d} s} .
$$

Note that

$$
\frac{\mathrm{d} p}{\mathrm{~d} t}(t) \leq(1-m+m v-m p(t)) p(t)
$$


We conclude that, for any sufficiently small $\epsilon>0$,

$$
p(t)<\frac{1-m}{m}+v+\epsilon
$$

holds for all large $t>0$. This establishes the ultimate uniform boundedness of solutions for (P).

Equation $(\mathrm{P})$ has two equilibria 0 and $v$. Denote by $p^{*}$ any of the equilibria. Then the linearization of $(\mathrm{P})$ at $p^{*}$ is given by

$$
\frac{\mathrm{d} p}{\mathrm{~d} t}(t)=\left[(1-3 m) p^{*}+m v\right] p(t)-(1-m) p^{*} p(t-\tau)
$$

If $p^{*}=0$, then it is unstable since (2.5) becomes

$$
\frac{\mathrm{d} p}{\mathrm{~d} t}(t)=m v p(t)
$$

If $p^{*}=v$, then the characteristic equation of (2.5) is

$$
\Delta(\lambda):=\lambda-(1-2 m) v+(1-m) v e^{-\lambda \tau}=0
$$

We investigate the dependence of local dynamics of $(\mathrm{P})$ on parameters $m$ and $\tau$.

Case 1. Choosing $m$ as parameter.

Define

$$
m_{k}=1-\frac{1}{2-\cos \eta_{k} \tau}
$$

where $\eta_{k}$ solves

$$
(2-\cos \eta \tau) \eta-v \sin \eta \tau=0,
$$

with the assumption of $v \tau>1$.

Proposition 2.1. If $m=1$, then $p^{*}=v$ is globally stable.

The case of $m=1$ represents that asset prices are totally determined by the fundamentalists. Obviously, asset prices cannot deviate from their fundamental values $v$ since the fundamentalists obey the EMH theory.

Theorem 2.2. Assume $v \tau>1$. Then

(i) the positive equilibrium $p^{*}=v$ of $(\mathrm{P})$ is asymptotically stable when $m \in\left(m_{0}, 1\right]$, where $m_{0}:=\max \left\{m_{k}\right\}$;

(ii) (P) undergoes a Hopf bifurcation at $v$ when $m=m_{k}$. 
Case 2. Regarding $\tau$ as parameter.

Define

$$
\tau_{j}=\frac{1}{\omega_{0}}\left(\arccos \frac{1-2 m}{1-m}+2 j \pi\right), \quad j=0,1,2, \ldots
$$

with $\omega_{0}:=v \sqrt{m(2-3 m)}$.

Theorem 2.3. For $(\mathrm{P})$,

(i) if $m \in[2 / 3,1]$, then $p^{*}=v$ is asymptotically stable for all $\tau \in \mathbb{R}^{+}$;

(ii) if $m \in(0,2 / 3)$, then $p^{*}=v$ is asymptotically stable when $0<\tau<\tau_{0}$ and becomes unstable when $\tau>\tau_{0}$; moreover, $(\mathrm{P})$ undergoes a Hopf bifurcation at $p^{*}=v$ when $\tau=\tau_{j}(j=$ $0,1,2, \ldots)$.

Theorem 2.4 gives the direction of Hopf bifurcation and stability of the bifurcating periodic solutions. Similar results hold if we choose $m$ as parameter.

Theorem 2.4. Assume $m \in(0,2 / 3)$. If $\operatorname{Re}\left(c_{1}(0)\right)<0(>0)$, then the bifurcating periodic solutions at $v$ are asymptotically stable (unstable) on the center manifold and the direction of bifurcation is forward (backward). In particular, the bifurcating periodic solution from the first bifurcation value $\tau=\tau_{0}$ is stable (unstable) in the phase space if $\operatorname{Re}\left(c_{1}(0)\right)<0(>0)$.

Corollary 2.5. When $\tau=\tau_{0}, v$ is stable (unstable) if $\operatorname{Re}\left(c_{1}(0)\right)<0(>0)$.

Remark 2.6. See the proof in Section 4 for the explanation of $c_{1}(0)$ which appears in Theorem 2.4 and Corollary 2.5 .

The conclusions above are illustrated in Figure 1, using bifurcation set on the $(m, \tau)$-plane. Here, $\tau_{0}(m), \tau_{1}(m), \tau_{2}(m), \ldots, \tau_{j}(m), \ldots$ are Hopf bifurcation curves. When

$$
(m, \tau) \in D:=\left\{(m, \tau) \mid 0<m<\frac{2}{3}, 0 \leq \tau<\tau_{0}(m)\right\} \cup\left\{(m, \tau) \mid \frac{2}{3} \leq m<1\right\}
$$

$p^{*}=v$ is asymptotically stable, and when

$$
(m, \tau) \in D^{c}:=\left\{(m, \tau) \mid 0<m<\frac{2}{3}, \tau>\tau_{0}(m)\right\}
$$

$p^{*}=v$ is unstable. Denote the curve $\tau=\tau_{0}(m)$ by $l$. Clearly, $l$ lies in the boundary of $D$. The stability of $v$ when $(m, \tau) \in l$ is given by Corollary 2.5 .

The occurrence of Hopf bifurcation explains the persistent deviation of the asset price from the fundamental value and it depends both on the time delay and the market fraction of the fundamentalists. In fact, choosing the same parameters as in [4, Figure 2]:

$$
m=0.62, \quad v=5, \quad \tau=2,
$$

we compute by (2.10) that $\tau_{0}=1.5304$. Therefore, Theorem 2.3 provides insight on the explanation of existence of a limit cycle in [4, Figure 2]. 


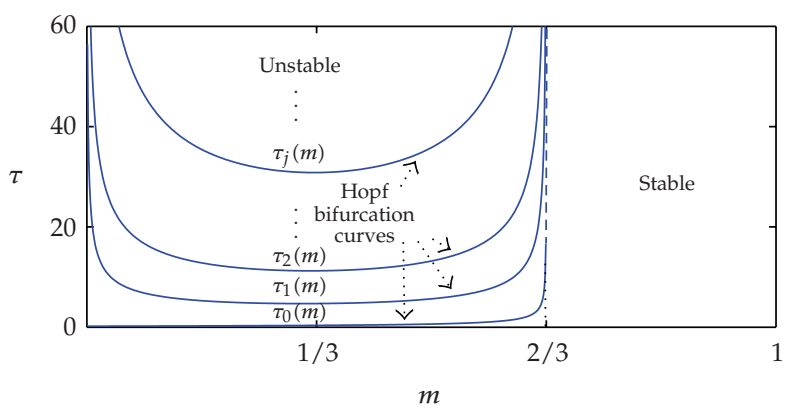

Figure 1: Hopf bifurcation set on $m-\tau$ plane.

Finally, a natural question is that if the bifurcating periodic solutions of $(\mathrm{P})$ exist when $\tau$ is far away from critical values? In the following theorem, we obtain the global continuation of periodic solutions bifurcating from the points $\left(v, \tau_{j}\right)(j=0,1,2, \ldots)$, using a global Hopf bifurcation theorem given by $\mathrm{Wu}[13]$.

Theorem 2.7. If $m \in(0,2 / 3)$, then for $\tau>\tau_{j}(j \geq 1)$, (P) has at least one periodic solution. Furthermore, if $m \in((3+\sqrt{2}) / 7,2 / 3)$, then $(\mathrm{P})$ has at least one periodic solution for $\tau>\tau_{j}(j \geq 0)$ and at least two periodic solutions for $\tau>\tau_{j}(j \geq 1)$. Here, $\tau_{j}(j=0,1,2, \ldots)$ is defined in (2.10).

Figures 2 and 3 describe the phenomena stated in Theorem 2.7, where $m=0.65 \in$ $((3+\sqrt{2}) / 7,2 / 3)$ and $v=5$. It is shown that the time delay plays an important role in the dynamics of the nonlinear model. To be concrete, the longer the time delays used by the chartists in estimating the asset price trend, the more likely the market will exhibit persistent deviation of the asset price from the fundamental value by a limit cycle, and the greater the period of a limit cycle becomes. In fact, $\tau_{0}=2.885$ and $c_{1}(0)=-0.856$ under these parameters. Therefore, combining Theorem 2.7 with the two figures, we know that there exists at least one stable periodic solution of $(\mathrm{P})$ with period $T(\tau) \geq 2 \pi / \omega=6.9706$ when $\tau>2.885$. We would like to mention that the graphs in Figure 2 are prepared by using DDE-BIFTOOL developed by Engelborghs et al. [20, 21].

\section{Proof of the Results in Cases 1 and 2}

Case 1. Choosing $m$ as parameter.

When $m=1,(\mathrm{P})$ is a scalar ordinary differential equation and therefore it has no nonconstant periodic solutions. Additionally, the only root of (2.7) is $\lambda=-v<0$. These complete the proof of Proposition 2.1.

When $m<1$, i $\eta(\eta>0)$ is a root of (2.7) if and only if $\eta$ satisfies

$$
\sin \eta \tau=\frac{\eta}{(1-m) v}, \quad \cos \eta \tau=\frac{1-2 m}{1-m} .
$$

It follows that

$$
\begin{gathered}
(2-\cos \eta \tau) \eta-v \sin \eta \tau=0, \\
m=1-\frac{1}{2-\cos \eta \tau} .
\end{gathered}
$$




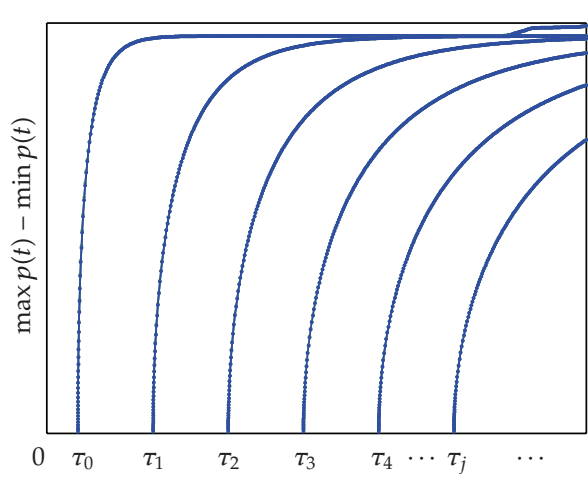

(a)

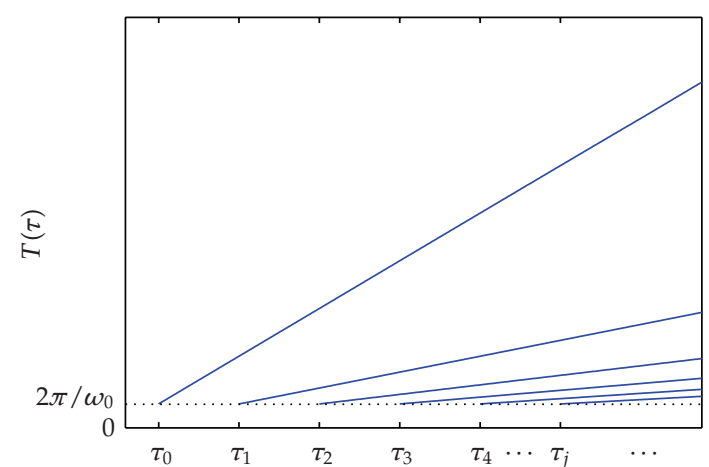

(b)

Figure 2: Hopf bifurcation branches through the centers $\left(v, \tau_{j}, 2 \pi / \omega_{0}\right)$. (a) on the $(\tau, d)$-plane, where $d=$ $\max p(t)-\min p(t) ;(\mathrm{b})$ on the $(\tau, T(\tau))$-plane, where $T(\tau)$ is the period of periodic solution bifurcated at $v$.

Denote $G(\eta) \stackrel{\text { def }}{=}(2-\cos \eta \tau) \eta-v \sin \eta \tau$. Then $G(0)=0, G(\infty)=\infty$, and

$$
G^{\prime}(\eta)=\eta \tau \sin \eta \tau+2-(1+v \tau) \cos \eta \tau
$$

Therefore, there exists at least one zero $\eta_{k}$ of $G(\eta)$ if $v \tau>1$.

Define $m_{k}$ as in (2.8), then $m_{k} \in(0,2 / 3)$ and $\pm i \eta_{k}$ is a pair of purely imaginary roots of (2.7) with $m=m_{k}$. Let $\lambda(m)$ be the root of (2.7) satisfying $\lambda\left(m_{k}\right)=i \eta_{k}$. Substituting $\lambda(m)$ into (2.7), it follows that

$$
\left(\frac{\mathrm{d} \lambda}{\mathrm{d} m}\right)=\frac{v e^{-\lambda \tau}-2 v}{1-\tau(1-m) v e^{-\lambda \tau}}
$$

Thus, we have the transversal condition

$$
\begin{aligned}
\left.\operatorname{Sign}\left(\operatorname{Re} \frac{\mathrm{d} \lambda}{\mathrm{d} m}\right)\right|_{m=m_{k}} & =\operatorname{Sign}\left\{\cos \eta_{k} \tau-2-\tau v\left(1-m_{k}\right)\left(1-2 \cos \eta_{k} \tau\right)\right\} \\
& =\operatorname{Sign}\left\{\frac{3 \tau v\left(1-m_{k}\right)^{2}-2 \tau v\left(1-m_{k}\right)-1}{1-m_{k}}\right\}, \\
& = \begin{cases}+1, & m_{k} \in\left(0, \frac{1}{3}\left(2-\sqrt{1+\frac{3}{\tau v}}\right)\right), \\
-1, & m_{k} \in\left(\frac{1}{3}\left(2-\sqrt{1+\frac{3}{\tau v}}\right), \frac{2}{3}\right),\end{cases}
\end{aligned}
$$

where $\cos \eta_{k} \tau=2-\left(1 / 1-m_{k}\right)$ is used. Accordingly, a Hopf bifurcation at $p^{*}=v$ occurs when $m=m_{k}$. 


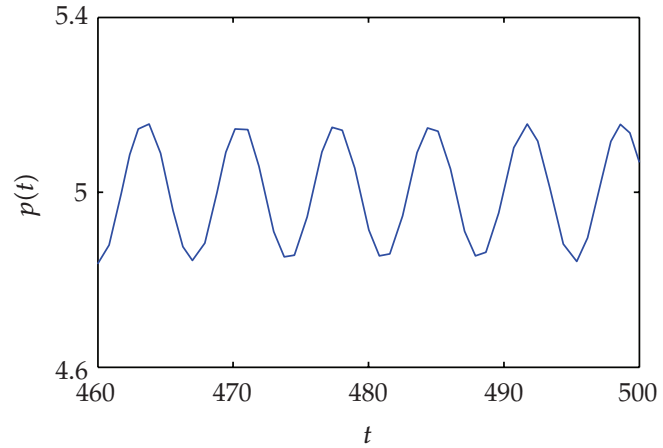

(a)

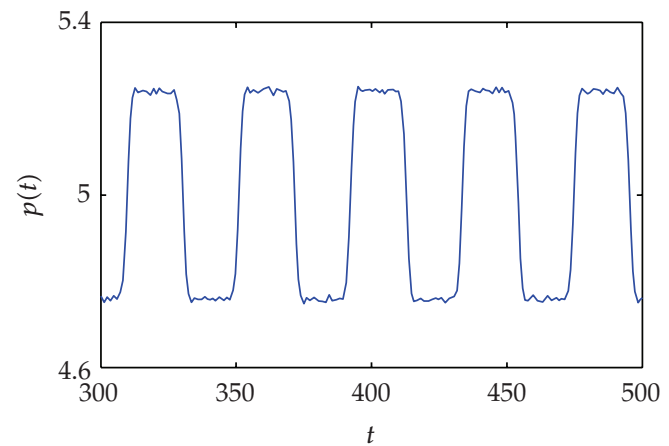

(c)

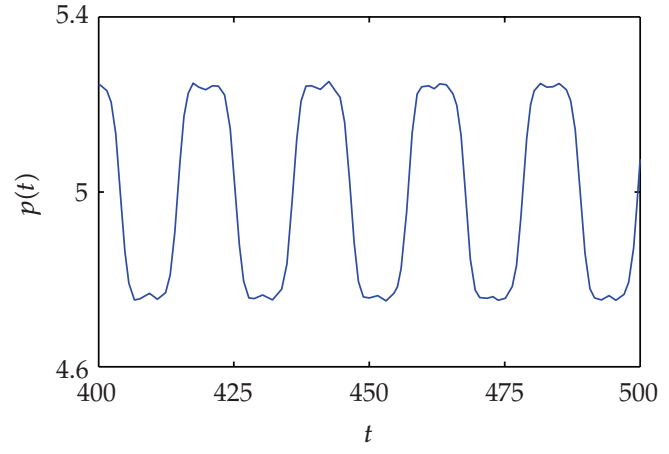

(b)

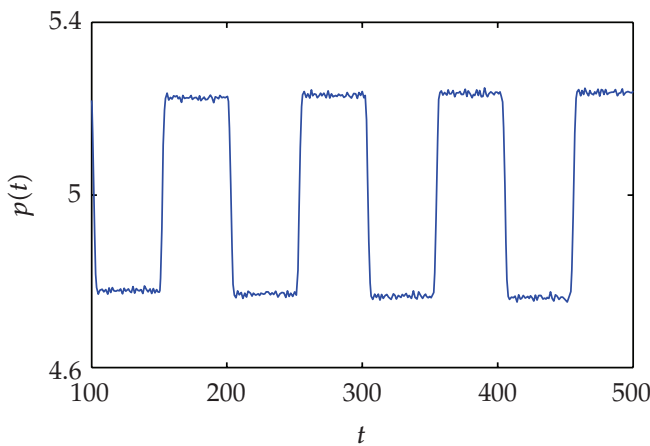

(d)

Figure 3: Stable periodic solution of (P), where (a) with $\tau=2.9>\tau_{0}=2.885$, (b) with $\tau=10$, (c) with $\tau=20$, and (d) with $\tau=50$.

In summary, if $v \tau>1$, then there exists at least one value of $m$ defined by (2.8) such that all the roots of $(2.7)$ have negative real parts when $m \in\left(m_{0}, 1\right)$, in addition, a pair of purely imaginary roots when $m=m_{k}$. This implies Theorem 2.2.

Case 2. Regarding $\tau$ as a parameter.

First of all, we know that the root of (2.7) with $\tau=0$ satisfies that $\lambda=-m v<0$. Therefore, $p^{*}=v$ is globally asymptotically stable when $\tau=0$.

Let $i \omega(\omega>0)$ be a root of (2.7). Then

$$
\sin \omega \tau=\frac{\omega}{(1-m) v}>0, \quad \cos \omega \tau=\frac{1-2 m}{1-m} .
$$

This leads to $\omega^{2}=m v^{2}(2-3 m) \cdot \omega_{0}=v \sqrt{m(2-3 m)}$ makes sense when $m \in(0,2 / 3)$. Define $\tau_{j}$ as in (2.10); then $i \omega_{0}$ is a pure imaginary root of (2.7) with $\tau=\tau_{j}, j=0,1,2, \ldots$.

Similarly, let $\lambda(\tau)=\alpha(\tau)+i \varrho(\tau)$ be the root of (2.7), satisfying

$$
\alpha\left(\tau_{j}\right)=0, \quad \rho\left(\tau_{j}\right)=\omega_{0} .
$$


Differentiating both sides of (2.7) gives

$$
\left(\frac{\mathrm{d} \lambda}{\mathrm{d} \tau}\right)^{-1}=\frac{e^{\lambda \tau}}{\lambda v(1-m)}-\frac{\tau}{\lambda}
$$

Therefore,

$$
\left.\operatorname{Re}\left(\frac{\mathrm{d} \lambda}{\mathrm{d} \tau}\right)^{-1}\right|_{\tau=\tau_{j}}=\frac{\sin \omega_{0} \tau_{j}}{\omega_{0} v(1-m)}>0 .
$$

This implies that $\alpha^{\prime}\left(\tau_{j}\right)>0, j=0,1,2, \ldots$

Note that $\lambda=0$ is not a root of (2.7) when $m=2 / 3$. Therefore, we obtain the following spectral properties of (2.7):

(i) if $m \in[2 / 3,1)$, then all roots of (2.7) have negative real parts;

(ii) if $m \in(0,2 / 3)$, then there exist a sequence values of $\tau$ defined by (2.10) such that (2.7) has a pair of purely imaginary roots $\pm i \omega_{0}$ when $\tau=\tau_{j}$. Additionally, all roots of (2.7) have negative real parts when $\tau \in\left[0, \tau_{0}\right)$, all roots of (2.7), except $\pm i \omega_{0}$, have negative real parts when $\tau=\tau_{0}$, and (2.7) has at least a pair of roots with positive real parts when $\tau>\tau_{0}$.

These spectral properties immediately lead to the dynamics near the positive equilibrium $v$ described in Theorem 2.3.

\section{Proof of Theorem 2.4}

We use the center manifold and normal form theories presented in Hassard et al. [12] to establish the proof of Theorem 2.4. Normalizing the delay $\tau$ by the time scaling $t \mapsto(t / \tau)$ and using the change of variables $p(t)=p(t \tau)$, we transform $(\mathrm{P})$ into

$$
\frac{\mathrm{d} p}{\mathrm{~d} t}(t)=(1-m) \tau \tanh (p(t)-p(t-1)) p(t)-m \tau(p(t)-v) p(t)
$$

whose characteristic equation at $p=v$ is

$$
\Delta_{1}(z):=z-(1-2 m) \tau v+(1-m) \tau v e^{-z}=0 .
$$

Comparing (4.1) with (2.7), we see $z=\lambda \tau$ for $\tau \neq 0$. Therefore, we obtain the following Lemma.

Lemma 4.1. Assume $m \in(0,2 / 3)$.

(i) If $\tau=\tau_{j}(j=0,1,2, \ldots)$, then (4.1) has a pair of purely imaginary roots $\pm i \omega_{0} \tau_{j}$, where $\tau_{j}$ and $\omega_{0}$ are defined as before. 
(ii) Let $z(\tau)=\gamma(\tau)+i \zeta(\tau)$ be the root of (4.1), satisfying

$$
\gamma\left(\tau_{j}\right)=0 \text { and } \zeta\left(\tau_{j}\right)=\omega_{0} \tau_{j}
$$

then

$$
\gamma^{\prime}\left(\tau_{j}\right)=\tau_{j} \alpha^{\prime}\left(\tau_{j}\right)>0, \quad j=0,1,2, \ldots
$$

(iii) All roots of (4.1) have negative real parts when $\tau \in\left[0, \tau_{0}\right)$, all roots of (4.1), except $\pm i \omega_{0} \tau_{0}$, have negative real parts when $\tau=\tau_{0}$, and (4.1) has at least a pair of roots with positive real parts when $\tau>\tau_{0}$.

Without loss of generality, we denote the critical value $\tau^{*}$ at which $\left(\mathrm{P}_{0}\right)$ undergoes a Hopf bifurcation at $v$. Let $\tau=\tau^{*}+\mu$, then $\mu=0$ is the Hopf bifurcation value of $\left(\mathrm{P}_{0}\right)$. Let $p_{1}(t)=p(t)-v$ and still denote $p_{1}(t)$ by $p(t)$, so that $\left(\mathrm{P}_{0}\right)$ can be written as

$$
\begin{aligned}
\frac{\mathrm{d} p}{\mathrm{~d} t}(t)= & v\left(\tau^{*}+\mu\right)[(1-2 m) p(t)-(1-m) p(t-1)] \\
& +\left(\tau^{*}+\mu\right)\left[(1-2 m) p^{2}(t)-(1-m) p(t) p(t-1)\right] \\
& -\frac{1}{3} v\left(\tau^{*}+\mu\right)(1-m)\left[p^{3}(t)-3 p^{2}(t) p(t-1)+3 p(t) p^{2}(t-1)-p^{3}(t-1)\right]+O(4) .
\end{aligned}
$$

For $\phi \in C([-1,0], \mathbb{R})$, define

$$
L_{\mu}(\phi)=v\left(\tau^{*}+\mu\right)[(1-2 m) \phi(0)-(1-m) \phi(-1)] .
$$

By the Riesz representation theorem, there exists a bounded variation function $\eta(\theta, \mu)(\theta \in$ $[-1,0])$ such that

$$
L_{\mu}(\phi)=\int_{-1}^{0}[\mathrm{~d} \eta(\theta, \mu)] \phi(\theta), \quad \text { for } \phi \in C([-1,0], \mathbb{R})
$$

In fact, we can choose

$$
\eta(\theta, \mu)= \begin{cases}v\left(\tau^{*}+\mu\right)(1-2 m), & \theta=0, \\ 0, & \theta \in(-1,0) \\ v\left(\tau^{*}+\mu\right)(1-m), & \theta=-1 .\end{cases}
$$


Define

$$
\begin{aligned}
A(\mu) \phi(\theta)= & \begin{cases}\frac{\mathrm{d} \phi(\theta)}{\mathrm{d} \theta}, & \theta \in[-1,0), \\
\int_{-1}^{0}[\mathrm{~d} \eta(\xi, \mu)] \phi(\xi), & \theta=0,\end{cases} \\
h(\mu, \phi)= & \left(\tau^{*}+\mu\right)\left[(1-2 m) \phi^{2}(0)-(1-m) \phi(0) \phi(-1)\right] \\
& -\frac{1}{3} v\left(\tau^{*}+\mu\right)(1-m)\left[\phi^{3}(0)-3 \phi^{2}(0) \phi(-1)+3 \phi(0) \phi^{2}(-1)-\phi^{3}(-1)\right]+O(4) .
\end{aligned}
$$

Furthermore, define the operator $R(\mu)$ as

$$
R(\mu) \phi(\theta)= \begin{cases}0, & \theta \in[-1,0), \\ h(\mu, \phi), & \theta=0 ;\end{cases}
$$

then (4.4) is equivalent to the following operator equation:

$$
\dot{x}_{t}=A(\mu) x_{t}+R(\mu) x_{t}
$$

where $x_{t}=x(t+\theta)$ for $\theta \in[-1,0]$.

For $\psi \in C^{1}([0,1], \mathbb{R})$, define an operator

$$
A^{*} \psi(s)= \begin{cases}-\frac{\mathrm{d} \psi(s)}{\mathrm{d} s}, & s \in(0,1], \\ \int_{-1}^{0} \psi(-\xi) \mathrm{d} \eta(\xi, 0), & s=0\end{cases}
$$

and a bilinear form

$$
\langle\psi(s), \phi(\theta)\rangle=\bar{\psi}(0) \phi(0)-\int_{-1}^{0} \int_{\xi=0}^{\theta} \bar{\psi}(\xi-\theta) \mathrm{d} \eta(\theta) \phi(\xi) \mathrm{d} \xi,
$$

where $\eta(\theta)=\eta(\theta, 0)$, then $A(0)$ and $A^{*}$ are adjoint operators.

From the preceding discussions, we know that $\pm i \omega_{0} \tau^{*}$ are eigenvalues of $A(0)$ and therefore they are also eigenvalues of $A^{*}$. It is not difficult to verify that the vectors $q(\theta)=$ $e^{i \omega_{0} \tau^{*} \theta}(\theta \in[-1,0])$ and $q^{*}(s)=\bar{l} e^{i \omega_{0} \tau^{*} s}(s \in[0,1])$ are the eigenvectors of $A(0)$ and $A^{*}$ corresponding to the eigenvalues $i \omega_{0} \tau^{*}$ and $-i \omega_{0} \tau^{*}$, respectively, where

$$
l=\left(1-v \tau^{*}(1-m) e^{-i \omega_{0} \tau^{*}}\right)^{-1}
$$


Following the algorithms stated in Hassard et al. [12], we obtain the coefficients which will be used in determining the important quantities:

$$
\begin{aligned}
g_{20} & =2 l \tau^{*}\left[(1-2 m)-(1-m) e^{-i \omega_{0} \tau^{*}}\right], \\
g_{11} & =l \tau^{*}\left[2(1-2 m)-(1-m)\left(e^{i \omega_{0} \tau^{*}}+e^{-i \omega_{0} \tau^{*}}\right)\right], \\
g_{02} & =2 l \tau^{*}\left[(1-2 m)-(1-m) e^{i \omega_{0} \tau^{*}}\right], \\
g_{21} & =2 l \tau^{*}(1-2 m)\left[2 W_{11}(0)+W_{20}(0)\right] \\
& -l \tau^{*}(1-m)\left[2 W_{11}(-1)+W_{20}(-1)+W_{20}(0) e^{i \omega_{0} \tau^{*}}+2 W_{11}(0) e^{-i \omega_{0} \tau^{*}}\right] \\
& -2 l \tau^{*} v(1-m)\left[3-3 e^{-i \omega_{0} \tau^{*}}-e^{i \omega_{0} \tau^{*}}+e^{-2 i \omega_{0} \tau^{*}}\right],
\end{aligned}
$$

where for $\theta \in[-1,0]$,

$$
\begin{aligned}
W_{20}(\theta) & =\frac{i g_{20}}{\omega_{0} \tau^{*}} q(\theta)+\frac{i \bar{g}_{02}}{3 \omega_{0} \tau^{*}} \bar{q}(\theta)+E_{1} e^{2 i \omega_{0} \tau^{*} \theta}, \\
W_{11}(\theta) & =-\frac{i g_{11}}{\omega_{0} \tau^{*}} q(\theta)+\frac{i \bar{g}_{11}}{\omega_{0} \tau^{*}} \bar{q}(\theta)+E_{2}, \\
E_{1} & =\frac{2\left[(1-2 m)-(1-m) e^{\left.-i \omega_{0} \tau^{*}\right]}\right.}{2 i \omega_{0}-v\left[(1-2 m)-(1-m) e^{-2 i \omega_{0} \tau^{*}}\right]}, \\
E_{2} & =\frac{2(1-2 m)-(1-m)\left(e^{i \omega_{0} \tau^{*}}+e^{-i \omega_{0} \tau^{*}}\right)}{m v} .
\end{aligned}
$$

Consequently, $g_{21}$ can be expressed explicitly.

Thus, we can compute the following values:

$$
\begin{aligned}
c_{1}(0) & =\frac{i}{2 \omega_{0} \tau^{*}}\left(g_{11} g_{20}-2\left|g_{11}\right|^{2}-\frac{\left|g_{02}\right|^{2}}{3}\right)+\frac{g_{21}}{2}, \\
\mu_{2} & =-\frac{\operatorname{Re}\left(c_{1}(0)\right)}{\operatorname{Re}\left(\lambda^{\prime}\left(\tau^{*}\right)\right)}, \\
\beta_{2} & =2 \operatorname{Re}\left(c_{1}(0)\right), \\
T_{2} & =-\frac{\operatorname{Im}\left(c_{1}(0)\right)+\mu_{2} \operatorname{Im}\left(\lambda^{\prime}\left(\tau^{*}\right)\right)}{\omega_{0} \tau^{*}},
\end{aligned}
$$

which determine the properties of bifurcating periodic solutions at the critical value $\tau^{*}$. More specifically, $\mu_{2}$ determines the directions of the Hopf bifurcation: if $\mu_{2}>0\left(\mu_{2}<0\right)$, then the bifurcating periodic solutions exist for $\tau>\tau^{*}\left(\tau<\tau^{*}\right) ; \beta_{2}$ determines the stability of bifurcating periodic solutions: the bifurcating periodic solutions on the center manifold are 
stable (unstable) if $\beta_{2}<0\left(\beta_{2}>0\right) ; T_{2}$ determines the period of the bifurcating periodic solutions: the period increases (decreases) if $T_{2}>0\left(T_{2}<0\right)$. Thus Theorem 2.4 follows.

In particular, when $\tau=\tau_{0}(m)$. It is well known that the normal form of the restriction of $\left(\mathrm{P}_{0}\right)$ with $\tau=\tau_{0}$ on the center manifold is given by

$$
\dot{z}(t)=i \omega_{0} \tau_{0} z+c_{1}(0) z^{2} \bar{z}+\cdots
$$

By Liapunov's second method, the zero solution of (4.17) is stable (unstable) if $\operatorname{Re}\left(c_{1}(0)\right)<$ $0(>0)$, and so is $v$.

\section{Proof of Theorem 2.7}

Assume $m \in(0,2 / 3)$. It is known that $(\mathrm{P})$ undergoes a local Hopf bifurcation at $p^{*}=v$ when $\tau=\tau_{j}(j=0,1, \ldots)$. Now we show that each bifurcation branch can be continued with respect to the parameter $\tau$ under the conditions in Theorem 2.7. We introduce the following notations:

$$
\begin{aligned}
X & =C([-\tau, 0], \mathbb{R}), \\
\Sigma & =C l\{(y, \tau, T): y \text { is a } T \text { - periodic solution of }(\mathrm{P})\} \subset X \times \mathbb{R}_{+} \times \mathbb{R}_{+}, \\
N & =\{(\widehat{y}, \tau, T): \hat{y}=0 \text { or } v\} .
\end{aligned}
$$

Let $C\left(v, \tau_{j}, 2 \pi / \omega_{0}\right)$ denote the connected component of $\left(v, \tau_{j}, 2 \pi / \omega_{0}\right)$ in $\Sigma$, and $\operatorname{Proj}_{\tau}\left(v, \tau_{j}, 2 \pi / \omega_{0}\right)$ its projection on $\tau$ component, where $\omega_{0}=v \sqrt{m(2-3 m)}$ and thus $\pm \mathrm{i} \omega_{0}$ is a pair of purely imaginary roots of (2.7) with $\tau=\tau_{j}$. By Theorem 2.3, we know that $C\left(v, \tau_{j}, 2 \pi / \omega_{0}\right)$ is nonempty.

Lemma 5.1. All bifurcating periodic solutions of $(\mathrm{P})$ from Hopf bifurcation at $v$ are positive if $\tau \in$ $\operatorname{Proj}_{\tau}\left(v, \tau_{j}, 2 \pi / \omega_{0}\right)$.

Proof. For each $\tau \in \operatorname{Proj}_{\tau}\left(v, \tau_{j}, 2 \pi / \omega_{0}\right)$, denote by $X(t, \tau)$ the corresponding nontrivial periodic solution, with its minimum value $\inf _{t \in \mathbb{R}^{+}} X(t, \tau)$. From the fact that the periodic solutions bifurcated from the positive equilibrium are continuous with respect to $\tau$ and $\lim _{\tau \rightarrow \tau_{j}} X(t, \tau)=v$ uniformly in $t \in \mathbb{R}$, we have that $\inf _{t \in \mathbb{R}} X(t, \tau)$ is continuous with respect to $\tau$, and the bifurcating periodic solutions are positive when $\tau$ varies in a small neighborhood of $\tau_{j}$. To prove the positivity, it is equivalent to prove $\inf _{t \in \mathbb{R}} X(t, \tau)>0$ when $\tau \in \operatorname{Proj}_{\tau}\left(v, \tau_{j}, 2 \pi / \omega_{0}\right)$. Otherwise, there exists a $\tau^{*} \in \operatorname{Proj}_{\tau}\left(v, \tau_{j}, 2 \pi / \omega_{0}\right)$ such that $\inf _{t \in \mathbb{R}} X\left(t, \tau^{*}\right)=0$. Without loss of generality, we assume that $\tau^{*}>\tau_{j}$ and $\inf _{t \in \mathbb{R}^{+}} X(t, \tau)>0$ when $\tau \in\left(\tau_{j}, \tau^{*}\right)$. By the proof of positivity of solution, we obtain $X\left(t, \tau^{*}\right) \equiv 0$. This implies that $\left(0, \tau^{*}, 2 \pi / \omega\right)$ is a center of $(\mathrm{P})$ for some $\omega>0$. This contradiction completes the proof.

Lemma 5.2. If $m \in(3+\sqrt{2} / 7,2 / 3)$, then $(\mathrm{P})$ has no positive periodic solution of period $4 \tau$.

Proof. Suppose that $y(t)$ is a positive periodic solution to $(\mathrm{P})$ of period $4 \tau$. Set

$$
u_{j}(t)=y(t-(j-1) \tau), \quad j=1,2,3,4
$$


Then $u(t)=\left(u_{1}(t), u_{2}(t), u_{3}(t), u_{4}(t)\right)$ is a periodic solution to the following system of ordinary differential equations:

$$
\begin{aligned}
& \dot{u}_{1}(t)=(1-m) \tanh \left(u_{1}-u_{2}\right) u_{1}-m\left(u_{1}-v\right) u_{1}, \\
& \dot{u}_{2}(t)=(1-m) \tanh \left(u_{2}-u_{3}\right) u_{2}-m\left(u_{2}-v\right) u_{2}, \\
& \dot{u}_{3}(t)=(1-m) \tanh \left(u_{3}-u_{4}\right) u_{3}-m\left(u_{3}-v\right) u_{3} \\
& \dot{u}_{4}(t)=(1-m) \tanh \left(u_{4}-u_{1}\right) u_{4}-m\left(u_{4}-v\right) u_{4}
\end{aligned}
$$

where $\cdot$ denotes $\mathrm{d} / \mathrm{d} t$. Let

$$
G=\left\{u \in \mathbb{R}: u_{j} \in\left(0, \frac{1-m}{m}+v\right], j=1,2,3,4\right\}
$$

For $(\mathrm{P})$, the ultimate uniform boundedness of solutions implies that the periodic solutions of (P) belong to the region $G$. To rule out the $4 \tau$-periodic solution to $(\mathrm{P})$, it suffices to prove the nonexistence of nonconstant periodic solutions of (5.3) in the region $G$. To do the latter, we use a general Bendixson's criterion in higher-dimensions developed by Li and Muldowney [19]. More specifically, we shall apply [19, Corollary 3.5]. The Jacobian matrix $J=J(u)$ of (5.3), for $u \in \mathbb{R}^{4}$, is

$$
J(u)=\left(\begin{array}{cccc}
A_{1} & B_{1} & 0 & 0 \\
0 & A_{2} & B_{2} & 0 \\
0 & 0 & A_{3} & B_{3} \\
B_{4} & 0 & 0 & A_{4}
\end{array}\right) .
$$

For $i=1,2,3,4$,

$$
\begin{gathered}
A_{i}=(1-m)\left[u_{i} \tanh ^{(1)}\left(u_{i}-u_{i+1}\right)+\tanh \left(u_{i}-u_{i+1}\right)\right]-2 m u_{i}+m v, \\
B_{i}=-(1-m) u_{i} \tanh ^{(1)}\left(u_{i}-u_{i+1}\right)<0,
\end{gathered}
$$

with $u_{5}=u_{1}$. The second additive compound matrix $J^{[2]}(u)$ of $J(u)$ is

$$
J^{[2]}(u)=\left(\begin{array}{cccccc}
A_{1}+A_{2} & B_{2} & 0 & 0 & 0 & 0 \\
0 & A_{1}+A_{3} & B_{3} & -B_{1} & 0 & 0 \\
0 & 0 & A_{1}+A_{4} & 0 & B_{1} & 0 \\
0 & 0 & 0 & A_{2}+A_{3} & B_{3} & 0 \\
-B_{4} & 0 & 0 & 0 & A_{2}+A_{4} & B_{2} \\
0 & -B_{4} & 0 & 0 & 0 & A_{3}+A_{4}
\end{array}\right) .
$$


Choose a vector norm in $\mathbb{R}^{6}$ as

$$
\left|\left(x_{1}, x_{2}, x_{3}, x_{4}, x_{5}, x_{6}\right)\right|=\max \left\{\sqrt{2}\left|x_{1}\right|,\left|x_{2}\right|, \sqrt{2}\left|x_{3}\right|, \sqrt{2}\left|x_{4}\right|,\left|x_{5}\right|, \sqrt{2}\left|x_{6}\right|\right\} .
$$

Then with respect to this norm, the Lozinskii measure $\mu\left(J^{[2]}(u)\right)$ of the matrix $J^{[2]}(u)$ is (see [22])

$$
\mu\left(J^{[2]}(u)\right)=\max \left\{A_{i}+A_{j}-\sqrt{2} B_{i}, A_{p}+A_{q}-\frac{\sqrt{2}}{2}\left(B_{p}+B_{q}\right)\right\},
$$

with $(i, j) \in\{(1,4),(2,1),(3,2),(4,3)\}$, and $(p, q) \in\{(1,3),(2,4)\}$. By [19, Corollary 3.5], system (5.3) has no periodic orbits in the region $G$ if $\mu\left(J^{[2]}(u)\right)<0$ for all $u \in G$. In fact, when $m \in(3+\sqrt{2} / 7,2 / 3)$, one can acquire the inequality as below,

$$
\mu\left(J^{[2]}(u)\right)<(1-m+m v)\left[2+\frac{2(1-3 m)+\sqrt{2}(1-m)}{m}\right]<0 .
$$

This completes the proof.

Lemma 5.3. Equation (P) has no nonconstant periodic solution of period $\tau$.

Proof. Equation $(\mathrm{P})$ has no nonconstant periodic solution of period $\tau$ is equivalent to the fact that $(\mathrm{P})$ with $\tau=0$ has no nonconstant periodic solution. It is straightforward that a first order autonomous ODE has no nonconstant periodic solutions. $(\mathrm{P})$ with $\tau=0$ is a first-order autonomous ODE, which proves the lemma.

Proof of Theorem 2.7. First note that for any $\tau_{j}$, the stationary points $\left(v, \tau_{j}, 2 \pi / \omega_{0}\right)$ of $(\mathrm{P})$ are nonsingular and isolated centers (see $\mathrm{Wu}[13]$ ) under the assumption $m \in(0,2 / 3)$; then the hypothesis $\left(\mathrm{H}_{2}\right)$ in [13] is satisfied. By (3.9), there exist $\epsilon>0, \delta>0$ and a smooth curve $\lambda:\left(\tau_{j}-\delta, \tau_{j}+\delta\right) \rightarrow \mathbb{C}$, such that

$$
\Delta(\lambda(\tau))=\Delta_{(v, \tau, T)}(\lambda(\tau))=0, \quad\left|\lambda(\tau)-i \omega_{0}\right|<\epsilon,
$$

for all $\tau \in\left[\tau_{j}-\delta, \tau_{j}+\delta\right]$, where $\Delta$ is defined as (2.7), and

$$
\lambda\left(\tau_{j}\right)=i \omega_{0},\left.\quad \frac{\mathrm{d} \operatorname{Re}(\lambda(\tau))}{\mathrm{d} \tau}\right|_{\tau=\tau_{j}}>0
$$

Denote $p_{j}=2 \pi / \omega_{0}$ and let

$$
\Omega_{\epsilon}=\left\{(0, q): 0<u<\epsilon,\left|q-p_{j}\right|<\epsilon\right\} .
$$


Clearly, if $\left|\tau-\tau_{j}\right| \leq \delta$ and $(u, q) \in \Omega_{\epsilon}$ such that $\Delta_{(v, \tau, T)}(u+2 \pi \mathrm{i} / q)=0$, then $\tau=\tau_{j}, u=0$, and $q=p_{j}$. Moreover, putting

$$
H^{ \pm}\left(v, \tau_{j}, \frac{2 \pi}{\omega_{0}}\right)(u, q)=\Delta_{\left(v, \tau_{j} \pm \delta, T\right)}\left(u+\mathrm{i} \frac{2 \pi}{q}\right)
$$

we have the crossing number

$$
\gamma_{1}\left(v, \tau_{j}, \frac{2 \pi}{\omega_{0}}\right)=\operatorname{deg}_{B}\left(H^{-}\left(v, \tau_{j}, \frac{2 \pi}{\omega_{0}}\right), \Omega \epsilon\right)-\operatorname{deg}_{B}\left(H^{+}\left(v, \tau_{j}, \frac{2 \pi}{\omega_{0}}\right), \Omega_{\epsilon}\right)=-1
$$

By the local Hopf bifurcation theorem for FDE [10], we conclude that the connected component $C\left(v, \tau_{j}, 2 \pi / \omega_{0}\right)$ through $\left(v, \tau_{j}, 2 \pi / \omega_{0}\right)$ in $\Sigma$ is nonempty. Meanwhile, we have

$$
\sum_{(\hat{y}, \tau, T) \in C\left(v, \tau_{j}, 2 \pi / \omega_{0}\right)} \gamma_{1}(\widehat{y}, \tau, T)<0
$$

and thus $C\left(v, \tau_{j}, 2 \pi / \omega_{0}\right)$ is unbounded by the global Hopf bifurcation theorem given by $\mathrm{Wu}$ [13].

Again, the property of ultimate uniform boundedness implies that the projection of $C\left(v, \tau_{j}, 2 \pi / \omega_{0}\right)$ onto the $y$-space is bounded. Meanwhile, (P) with $\tau=0$ has no nonconstant periodic solutions since it is a first-order autonomous ordinary differential equation. Therefore, $\operatorname{Proj}_{\tau}\left(v, \tau_{j}, 2 \pi / \omega_{0}\right)$ is bounded below.

By the definition of $\tau_{j}$ given in (2.10), we know that, when $m \in(0,2 / 3)$,

$$
2 \pi<\tau_{j} \omega_{0}<2(j+1) \pi, \quad j \geq 1,
$$

which implies that

$$
\frac{\tau_{j}}{j+1}<\frac{2 \pi}{\omega_{0}}<\tau_{j}
$$

By Lemma 5.3, we have $\tau /(j+1)<T<\tau$ if $(x, \tau, T) \in C\left(v, \tau_{j}, 2 \pi / \omega_{0}\right)$ for $j \geq 1$. Because $C\left(v, \tau_{j}, 2 \pi / \omega_{0}\right)$ is unbounded, $\operatorname{Proj}_{\tau}\left(v, \tau_{j}, 2 \pi / \omega_{0}\right)$ must be unbounded. Consequently, $\operatorname{Proj}_{\tau}\left(v, \tau_{j}, 2 \pi / \omega_{0}\right)$ include $\left[\tau_{j}, \infty\right)$ for $j \geq 1$. The former part of the theorem follows.

Moreover, if $m \in((3+\sqrt{2}) / 7,2 / 3)$, then

$$
\frac{\pi}{2}<\tau_{0} \omega_{0}<\pi
$$

where (3.6) is used. This implies that

$$
2 \tau_{0}<\frac{2 \pi}{\omega_{0}}<4 \tau_{0}
$$


Thus, we have by Lemma 5.2 that $2 \tau<T<4 \tau$ if $(x, \tau, T) \in C\left(v, \tau_{0}, 2 \pi / \omega_{0}\right)$. Similarly, this shows that in order for $C\left(v, \tau_{0}, 2 \pi / \omega_{0}\right)$ to be unbounded, $\operatorname{Proj}_{\tau}\left(v, \tau_{0}, 2 \pi / \omega_{0}\right)$ must be unbounded. This implies that $\operatorname{Proj}_{\tau}\left(v, \tau_{0}, 2 \pi / \omega_{0}\right)$ includes $\left[\tau_{0}, \infty\right)$.

In addition, the first global Hopf branch contains periodic solutions with period between $2 \tau$ and $4 \tau$, which are the slowly oscillating periodic solutions. The $j$ th branches, for $j \geq 1$, since the periods are less than $\tau$, contain fast-oscillating periodic solutions. Therefore, (P) has at least two periodic solutions for $\tau>\tau_{0}$ provided $m \in((3+\sqrt{2)} / 7,2 / 3)$. The proof of Theorem 2.7 is complete.

\section{Acknowledgments}

This research is supported by the NNSF (no. 10771045) of China, the Program of Excellent Team in HIT and the Natural Science Foundation of Heilongjiang Province, (A200806). The authors are grateful to Professor Xuezhong He and the anonymous referees for their helpful comments and valuable suggestions.

\section{References}

[1] C. Chiarella, R. Dieci, and L. Gardini, "Speculative behaviour and complex asset price dynamics: a global analysis," Journal of Economic Behavior and Organization, vol. 49, no. 2, pp. 173-197, 2002.

[2] C. Chiarella, R. Dieci, and X. He, "Heterogeneity, market mechanisms and asset price dynamics," in Handbook of Financial Markets: Dynamics and Evolution, chapter 5, pp. 277-344, 2009.

[3] C. Chiarella and X.-Z. He, "Heterogeneous beliefs, risk, and learning in a simple asset-pricing model with a market maker," Macroeconomic Dynamics, vol. 7, no. 4, pp. 503-536, 2003.

[4] G. Dibeh, "Speculative dynamics in a time-delay model of asset prices," Physica A, vol. 355, no. 1, pp. 199-208, 2005.

[5] X.-Z. He, K. Li, J. Wei, and M. Zheng, "Market stability switches in a continuous-time financial market with heterogeneous beliefs," Economic Modelling, vol. 26, no. 6, pp. 1432-1442, 2009.

[6] C. H. Hommes, "Financial markets as nonlinear adaptive evolutionary systems," Quantitative Finance, vol. 1, no. 1, pp. 149-167, 2001.

[7] C. Hommes, "heterogeneous agent models in economics and finance," in Handbook of Computational Economics, vol. 2, chapter 23, pp. 1109-1186, 2006.

[8] T. Lux, "The socio-economic dynamics of speculative markets: interacting agents, chaos, and the fat tails of return distributions," Journal of Economic Behavior and Organization, vol. 33, no. 2, pp. 143-165, 1998.

[9] R. Sethi, "Endogenous regime switching in speculative markets," Structural Change and Economic Dynamics, vol. 7, no. 1, pp. 99-118, 1996.

[10] J. K. Hale and S. M. V. Lunel, Introduction to Functional-Differential Equations, vol. 99 of Applied Mathematical Sciences, Springer, New York, NY, USA, 1993.

[11] J. Carr, Applications of Centre Manifold Theory, vol. 35 of Applied Mathematical Sciences, Springer, New York, NY, USA, 1981.

[12] B. D. Hassard, N. D. Kazarinoff, and Y. H. Wan, Theory and Applications of Hopf Bifurcation, vol. 41 of London Mathematical Society Lecture Note Series, Cambridge University Press, Cambridge, UK, 1981.

[13] J. H. Wu, "Global continua of periodic solutions to some difference-differential equations of neutral type," The Tohoku Mathematical Journal, vol. 45, no. 1, pp. 67-88, 1993.

[14] J. Wei, "Bifurcation analysis in a scalar delay differential equation," Nonlinearity, vol. 20, no. 11, pp. 2483-2498, 2007.

[15] J. Wei and D. Fan, "Hopf bifurcation analysis in a Mackey-Glass system," International Journal of Bifurcation and Chaos, vol. 17, no. 6, pp. 2149-2157, 2007.

[16] J. Wei and M. Y. Li, "Hopf bifurcation analysis in a delayed Nicholson blowflies equation," Nonlinear Analysis: Theory, Methods \& Applications, vol. 60, no. 7, pp. 1351-1367, 2005.

[17] J. Wei and C. Yu, "Hopf bifurcation analysis in a model of oscillatory gene expression with delay," Proceedings of the Royal Society of Edinburgh: Section A, vol. 139, no. 4, pp. 879-895, 2009. 
[18] J. H. Wu, "Symmetric functional-differential equations and neural networks with memory," Transactions of the American Mathematical Society, vol. 350, no. 12, pp. 4799-4838, 1998.

[19] Y. Li and J. S. Muldowney, "On Bendixson's criterion," Journal of Differential Equations, vol. 106, no. 1, pp. 27-39, 1993.

[20] K. Engelborghs, T. Luzyanina, and D. Roose, "Numerical bifurcation analysis of delay differential equations using DDE-BIFTOOL," Association for Computing Machinery, vol. 28, no. 1, pp. 1-21, 2002.

[21] K. Engelborghs, T. Luzyanina, and G. Samaey, "DDE-BIFTOOL v. 2.00: a matlab package for bifurcation analysis of delay differential equations," Technical Report TW-330, Department of Computer Science, K. U. Leuven, Leuven, Belgium, 2001.

[22] W. A. Coppel, Stability and Asymptotic Behavior of Differential Equations, D. C. Heath, Boston, Mass, USA, 1965. 


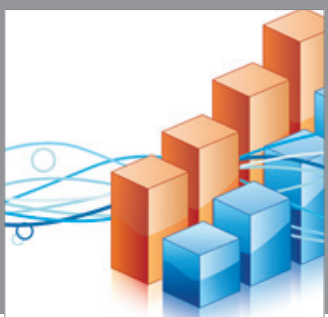

Advances in

Operations Research

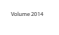

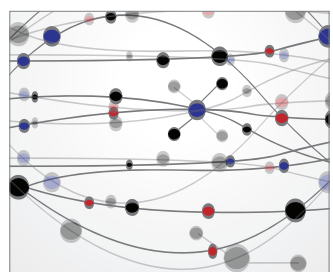

\section{The Scientific} World Journal
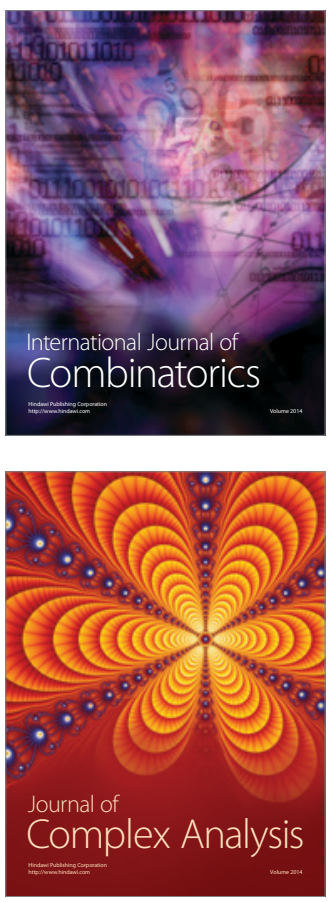

International Journal of

Mathematics and

Mathematical

Sciences
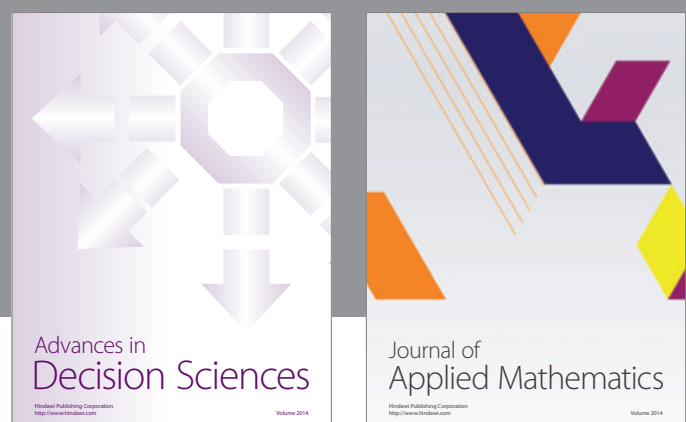

Journal of

Applied Mathematics
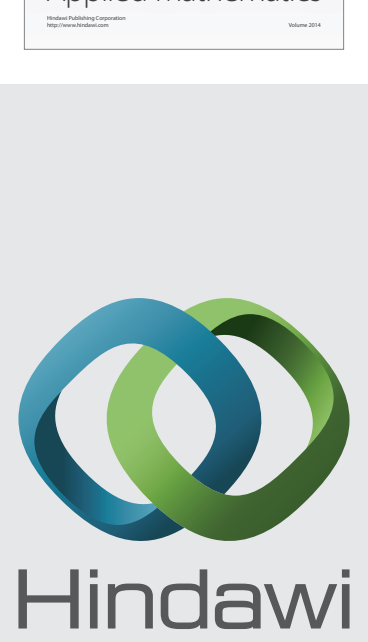

Submit your manuscripts at http://www.hindawi.com
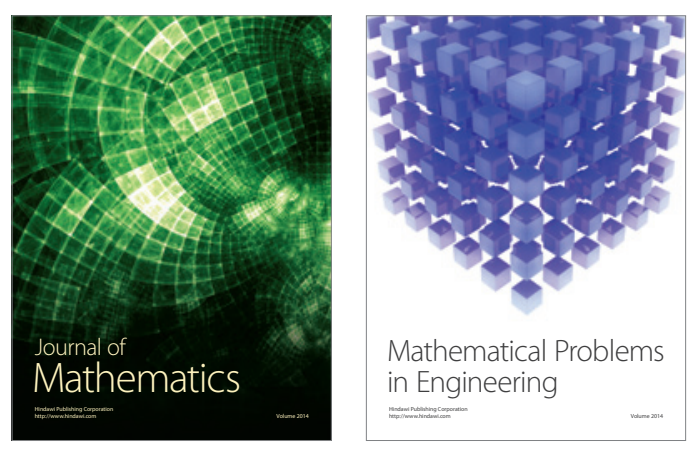

Mathematical Problems in Engineering
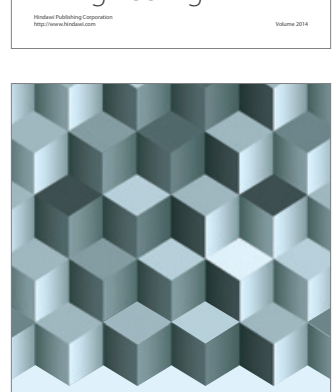

Journal of

Function Spaces
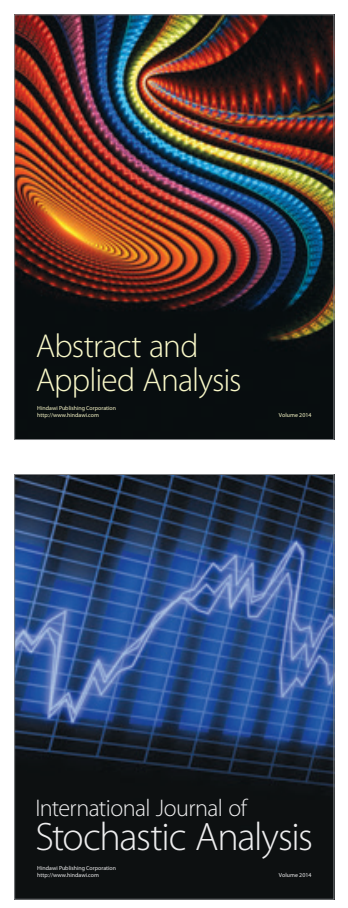

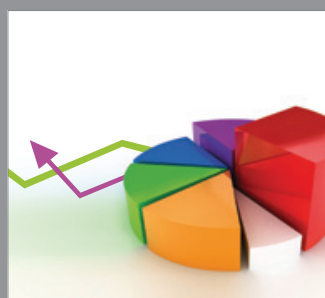

ournal of

Probability and Statistics

Promensencen
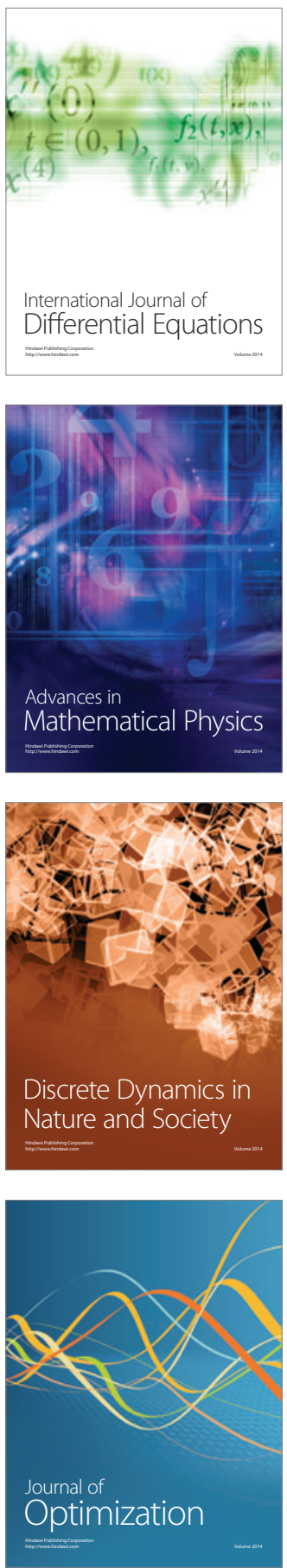